
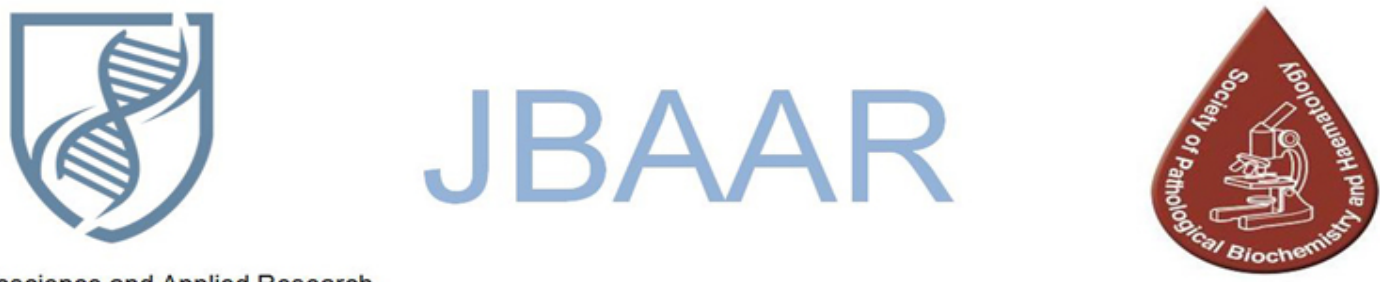

\title{
The Egyptian population records significant differences in allele frequencies as compared to other Arabian populations from Morocco, Syria and Gaza using short tandem repeat system
}

\author{
Samir A. Nassar ${ }^{1}$, Nermien A. Ibrahim ${ }^{2}$, Ali M. Abd El-Gawad ${ }^{3}$ and Mohamed F. Omar ${ }^{4}$ \\ 1,3,4: Department of Zoology, Faculty of Science, Zagazig University, Egypt. \\ 2: Department of Forensic Medicine and Clinical Toxicology, Faculty of Medicine, Zagazig University, Egypt. \\ (Corresponding author e-mail samnas59@yahoo.com)
}

\begin{abstract}
The allele frequencies of the selected somatic short tandem repeat (STR) polymorphic loci (D3S1358, TH01, D21S11, D18S51, Penta E, D5S818, D13S317, D7S820, D16S539, CSF1PO, Penta D, vWA, D8S1179, TPOX and FGA ) were evaluated for 103 buccal swab samples of healthy unrelated Egyptian individuals collected from 27 governorates. Two DNA extraction methods were used (a manual organic one and BioRobot EZ1 automatic method). Samples were amplified by PowerPlex ${ }^{\mathrm{R}} 16$ system, genotyped and allele frequencies were calculated. The aim of this work was to establish a STR database for 15 loci in the Egyptian population and comparing their DNA characteristics with those of Morocco, Syria and Gaza, located at the very West and East of Mediterranean sea, respectively, and to chick if they can be fused to a single Arabian database. The present results indicated that buccal cell DNA samples can provide precise estimates of human amplifiable DNA. DNA yields obtained with the BioRobot EZ1 were of better quantity and quality of extracted DNA. In the studied Egyptian population, the current investigation recorded allele specificity in some loci; locus D21S11 (allele 28.2) and locus D18S51 (allele 13.2). However, other alleles could be recorded as non-specific for the Egyptian population like allele 16 of Penta D locus and alleles 6 and 13 of TPOX locus. The interpopulation comparison between the obtained Egyptian population database and the previously mentioned Arabian populations revealed significant differences allover the 15 loci. The presence of allele 20 of D3S1358 locus, allele 24.2 of D21S11 locus and allele19.2 of FGA locus in a sample from a crime scene and it is

referred to unknown person indicates that the person is not Egyptian but from Morocco. If the allele 35.2 of D21S11 locus, allele 17.2 of D18S51 locus, allele 5 of CSF1PO and allele 9 of FGA locus is found in a sample from a crime scene and it referred to an unknown person, this indicates that the person is not Egyptian but from Gaza . If allele 16.4 of Penta E locus, allele 7 of D13S317 locus, allele 9.3 of D7S820 locus, allele 15 of D16S539 locus and allele 11.3 of D8S1179 locus found in a sample from a crime scene and it is referred to an unknown person, this indicates that the person is not Egyptian but from Syria. Thus, a common STR database for the Arabian population cannot be established. In conclusion, the present data may be considered as a helpful preparation for a preliminary construction of the local DNA database of the Egyptian population.

Keywords: allele frequencies, Egyptian population, Arabian populations, short tandem repeat system

\section{Introduction}

Deoxyribonucleic acid (DNA) is the molecule of life. It is the chemical code that is found in every cell of an individual's body specifying his function, appearance and pedigree. It is unique for all individuals except identical twins. An individual DNA is formed by a combination of DNA from his or her parents with half coming from the mother and half from the father. Because it is unique the ability to examine DNA found at a crime scene is very useful forensic tool. Forensic science utilizes the properties of DNA in several ways Watson and Crick (1953). National DNA databases have become one of the most efficient tools
\end{abstract}


to provide intelligence about unknown perpetrators in criminal investigations.(ENFSI DNA Working Group). STRs have become widely used in human identification, particularly in criminal cases and in mass disasters. In such cases the substrates for the analyses may be decomposed biological material (Carvalho et al., 2009). Pure genomic DNA extraction from biological samples is the vital primary step to succeed in various molecular biological techniques such as polymerase chain reaction (PCR), restriction enzyme analysis, mutation detection, and genotyping as well as linkage analysis (Phillips et al., 2000 and Wang et al., 2003). The quality of DNA extracted from buccal cells may be related to the subsequent PCR. Longterm storage may lead to degradation of human DNA. Generally, the laboratory is not always available for sample processing immediately after collection in a field study. Therefore, sample storage is frequently necessary. However, the time of collection and storage duration influence the quality of DNA extracted (Nedel et al., 2009). The quantity and quality of DNA from saliva, even after a period in storage, and the techniques employed are adequate for forensic DNA analysis. (Carvalho et al., 2010). The use of the Bio Robot EZ1 in the forensic casework has streamlined into the DNA analysis by reducing the need for labor-intensive phenol chloroform extraction (Monopetit et al., 2005). The use of DNA evidence and convicted offender DNA databases has expanded significantly since the first U.S. DNA database was created (Ashcroft et al., 2002).. There is an ongoing need for greater public and policy debate as DNA databases expand around the world. Some safeguards are implemented at the national or regional level, but there is an ongoing lack of global standards and a need for more societal engagement and debate, National DNA databases have become one of the most efficient tools to provide intelligence about unknown perpetrators in criminal investigations. (Wallace et al., 2014).

The aim of this work was to establish a STR database for 15 STR loci in the Egyptian population and comparing their DNA characteristics with those of Morocco, Syria and Gaza.

\section{Materials and Methods}

The population samples have been collected from 103 unrelated persons from both sexes and from 27 Egyptian governorates using buccal swaps. Two DNA extraction methods were used, the first is manual (Organic extraction method) and the second is automatic (BioRobot ${ }^{\circledR}$ EZ1extraction method) to extract 103 samples and then measure the concentration of DNA of each sample by using NanoDrop ND-1000 spectrophotometer (NanoDrop Technologies Inc., Wilmington, Delaware, USA), and determination the mean of all concentrations of each extraction method. The amplification of samples was carried out by PowerPlex ${ }^{\circledR} 16$ HS system from (Promega) using polymerase chain reaction (PCR) volumes in a GeneAmp 9600 (Applied Biosystems). PCR products were separated by capillary gel electrophoresis on an ABI
PRISM 310 Genetic Analyzer with laser-induced fluorescence (LIF) detection. According to the statistical parameters the combined analysis of these 15 STR loci is a powerful tool for forensic identification for the Arabian populations.

\section{Results and Discussion}

\subsection{Extraction and concentration of DNA}

The extraction of DNA using organic extraction method (Phenol Chloro Isoamyl alcohol mixture) yielded high amount of DNA (31.28 mg/ml). The extraction of DNA using BioRobot ${ }^{\circledR}$ EZ1 EZ1extraction method. yielded a moderate amount of DNA $(15.58 \mathrm{mg} / \mathrm{ml})$. The current study proved that Biorobot EZ1 method was the best method for better quantity and quality of extracted DNA .

\subsection{Allele frequency for 103 unrelated persons from} Egyptian population:

Table (1) disclosed the characteristics of DNA genotyping and allele frequancies in the Egyptian population as follow: at D3S1358 locus there is no allele have the number 12 or 20 and it was polymorphic with the alleles 17, 16 and 15 most frequent (at $0.286,0.271$ and 0.247 respectively). At THO1 locus there is no allele have the number4, 5,11 and 13.3 and it was polymorphic with the alleles 9, 6 and 7 most frequent (at $0.368,0.208$ and 0.174 respectively). At D21S11 locus there is no allele have the number 24, 24.2, 25, 25.2, 34, 34.2, 35.2, 36, 37 and 38 and it was polymorphic with the alleles 30, 29 and 28 most frequent (at $0.262,0.218$ and 0.116 respectively). At D18S51 locus there is no allele have the number $8,9,10,10.2,22,23,24$, 25, 26 and 27 and it was polymorphic with the alleles 12 , 13 and 14 most frequent (at 0.174, 0.169 and 0.140 respectively). At Penta E locus there is no allele have the number 6, 22, 23 and 24 and it was polymorphic with the alleles 12,7 and 11 most frequent (at 0.179, 0.135 and 0.116 respectively). At D5S818 locus there is no allele have the number 16 and it was polymorphic with the alleles 12, 11 and 13 most frequent (at $0.359,0.223$ and 0.174 respectively). At D13S317 locus there is no allele have the number 7 and 15 and it was polymorphic with the alleles 12, 11 and 8 most frequent (at 0.388, 0.242 and 0.135 respectively). At D7S820 locus there is no allele have the number 6 and it was polymorphic with the alleles 10, 11 and 8 most frequent (at 0.305, 0.237 and 0.218 respectively). At D16S539 locus there is no allele have the number5 and 15 and it was polymorphic with the alleles 11, 12 and 13 most frequent (at 0.339, 0.203 and 0.160 respectively). At CSF1PO locus there is no allele have the number 6 and 15 and it was polymorphic with the alleles 10, 12 and 11 most frequent (at 0.334, 0.296 and 0.271 respectively). In Egyptian population at Penta D locus there is no allele have the number 16 and 17 and it was polymorphic with the alleles 11, 9 and 10 most frequent (at $0.223,0.203$ and 0.174 respectively). At vWA locus there is no allele have the number 10,11, 12, 13 and 22 and it was polymorphic with the alleles 17,16 and 18 most frequent (at $0.315,0.208$ and 0.179 respectively). At D8S1179 locus there is no allele have the number 7 and 18 
Table 1: Allele frequencies for the 15 STR loci in the Egyptian population (103 Sample.)

\begin{tabular}{|c|c|c|c|c|c|c|c|c|c|c|c|c|c|c|c|}
\hline Allele & D3S1358 & TH01 & D21S11 & D18S51 & Penta E & D5S818 & D13S317 & D7S820 & D16S539 & CSF1PO & Penta D & vWA & D8S1179 & TPOX & FGA \\
\hline 2.2 & & & & & & & & & & & 0.029 & & & & \\
\hline 3.2 & & & & & & & & & & & 0.004 & & & & \\
\hline 5 & & & & & 0.019 & & & & & & 0.009 & & & & \\
\hline 6 & & \begin{tabular}{ll|}
0.208 \\
\end{tabular} & & & & & & & & & & & & & \\
\hline 7 & & $\begin{array}{ll}0.174 \\
\end{array}$ & & & 0.135 & 0.019 & & \begin{tabular}{l|l|l}
0.004 \\
\end{tabular} & & 0.004 & \begin{tabular}{|c|c|}
0.009 \\
\end{tabular} & & & 0.009 & \\
\hline 8 & & 0.140 & & & 0.067 & 0.053 & 0.135 & 0.218 & 0.038 & 0.033 & \begin{tabular}{|c|}
0.029 \\
\end{tabular} & & 0.019 & 0.470 & \\
\hline 9 & & $\begin{array}{l}0.368 \\
\end{array}$ & & & $\begin{array}{l}0.004 \\
\end{array}$ & 0.053 & $\begin{array}{ll}0.063 \\
\end{array}$ & 0.111 & 0.135 & 0.009 & \begin{tabular}{|l|l|}
0.203 \\
\end{tabular} & & 0.004 & 0.179 & \\
\hline 9.3 & & $\begin{array}{l}0.058 \\
\end{array}$ & & & & & & & & & & & & & \\
\hline 10 & & $\begin{array}{l}0.048 \\
\end{array}$ & & & $\begin{array}{l}0.053 \\
\end{array}$ & 0.087 & 0.053 & 0.305 & 0.101 & 0.334 & \begin{tabular}{|l|l|}
0.174 \\
\end{tabular} & & 0.063 & 0.067 & \\
\hline 11 & & & & 0.009 & 0.116 & 0.223 & 0.242 & 0.237 & 0.339 & 0.271 & \begin{tabular}{|c|c|}
0.223 \\
\end{tabular} & & 0.092 & 0.223 & \\
\hline 12 & & & & 0.174 & 0.179 & 0.359 & $\begin{array}{l}0.388 \\
\end{array}$ & \begin{tabular}{c|}
0.092 \\
\end{tabular} & 0.203 & 0.296 & \begin{tabular}{|l|l|}
0.116 \\
\end{tabular} & & 0.121 & \begin{tabular}{l|l}
0.048 \\
\end{tabular} & \\
\hline 13 & 0.004 & & & 0.169 & 0.111 & 0.174 & $\mathbf{0 . 0 7 7}$ & 0.019 & 0.160 & 0.043 & \begin{tabular}{|l|l|}
0.121 \\
\end{tabular} & & 0.189 & & \\
\hline 13.2 & & & & 0.004 & & & & & & & & & & & \\
\hline 14 & 0.067 & & & 0.140 & $\begin{array}{l}0.053 \\
\end{array}$ & 0.024 & \begin{tabular}{|l|l}
0.038 \\
\end{tabular} & \begin{tabular}{c|}
0.009 \\
\end{tabular} & 0.019 & 0.004 & \begin{tabular}{|l|l|}
0.048 \\
\end{tabular} & 0.058 & 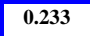 & & \\
\hline 15 & 0.247 & & & 0.121 & 0.063 & 0.004 & & & & & \begin{tabular}{|c|}
0.029 \\
\end{tabular} & 0.135 & 0.194 & & \\
\hline 16 & 0.271 & & & 0.121 & $\begin{array}{l}0.072 \\
\end{array}$ & & & & & & & 0.208 & 0.077 & & \\
\hline 17 & 0.286 & & & 0.082 & 0.033 & & & & & & & 0.315 & \begin{tabular}{|l|l|}
0.004 \\
\end{tabular} & & \\
\hline 17.2 & & & & & & & & & & & & & & & \\
\hline 18 & 0.116 & & & 0.116 & $\begin{array}{l}0.038 \\
\end{array}$ & & & & & & & 0.179 & & & \\
\hline 19 & 0.004 & & & $\begin{array}{l}0.029 \\
\end{array}$ & 0.019 & & & & & & & 0.092 & & & 0.063 \\
\hline 20 & & & & 0.019 & 0.024 & & & & & & & 0.004 & & & 0.077 \\
\hline 21 & & & & 0.009 & 0.004 & & & & & & & 0.004 & & & 0.150 \\
\hline 21.2 & & & & & & & & & & & & & & & 0.004 \\
\hline 22 & & & & & & & & & & & & & & & 0.121 \\
\hline 23 & & & & & & & & & & & & & & & 0.194 \\
\hline 24 & & & & & & & & & & & & & & & 0.184 \\
\hline 25 & & & & & & & & & & & & & & & 0.092 \\
\hline 26 & & & 0.004 & & & & & & & & & & & & 0.053 \\
\hline 27 & & & 0.029 & & & & & & & & & & & & 0.014 \\
\hline 28 & & & 0.116 & & & & & & & & & & & & 0.024 \\
\hline 28.2 & & & 0.004 & & & & & & & & & & & & \\
\hline 29 & & & 0.218 & & & & & & & & & & & & 0.014 \\
\hline 29.2 & & & 0.004 & & & & & & & & & & & & \\
\hline 30 & & & 0.262 & & & & & & & & & & & & 0.004 \\
\hline 30.2 & & & 0.004 & & & & & & & & & & & & \\
\hline 31 & & & 0.067 & & & & & & & & & & & & \\
\hline 31.2 & & & $\begin{array}{l}0.106 \\
\end{array}$ & & & & & & & & & & & & \\
\hline 32 & & & $\begin{array}{l}.019 \\
\end{array}$ & & & & & & & & & & & & \\
\hline 32.2 & & & 0.111 & & & & & & & & & & & & \\
\hline 33 & & & 0.004 & & & & & & & & & & & & \\
\hline 33.2 & & & 0.033 & & & & & & & & & & & & \\
\hline 35 & & & 0.004 & & & & & & & & & & & & \\
\hline
\end{tabular}


most frequent (at $0.315,0.208$ and 0.179 respectively). At D8S1179 locus there is no allele have the number 7 and 18 and it was polymorphic with the alleles 14, 15 and 13 most frequent (at $0.233,0.194$ and 0.189 respectively). At TPOX locus there is no allele have the number 6 and 13 and it was polymorphic with the alleles 8, 11 and 9 most frequent (at $0.470,0.223$ and 0.179 respectively). At FGA locus there is no allele have the number $16,17,18,18.2,19.2,20.2,22.2$, 23.2, 24.2, 25.2, 31.2, 43.2, 44.2, 45.2 and 46.2 it was polymorphic with the alleles 23, 24 and 21 most frequent (at $0.194,0.184$ and 0.150 respectively).

Concerning the specificity of the DNA characteristics of the The Egyptian population allele 13.2 of D18S51 locus and allele 21 at vWA locus is specific for Egyptian population but was not appears in the three other populations while allele 16 at Penta D locus and alleles 6 \& 13 is not specific for Egyptian population.

3.3. Comparison between Allele frequencies of Egyptian Population to other Arabian populations :

The comparison was made between Egyptian population database and three other populations from Syria \& Morocco (Abdin., et al 2003) and Palestinian population (Masoud, 2008).

The presence of the allele 20 of D3S1358 locus, the allele 24.2 of D21S11 locus and the allele 19.2 of FGA locus found in a sample from crime scene and it is referred to unknown person, this indicates that the person is not Egyptian but from Morocco.

If the allele 35.2 of D21S11 locus, the allele 17.2 of D18S51 locus, the allele 5 of CSF1PO and the allele 9 of FGA locus found in a sample from crime scene and it is referred to an unknown person, This indicates that the person is not Egyptian but from Gaza.

If the allele 16.4 of Penta E locus, the allele 7 of D13S317 locus, the allele 9.3 of D7S820 locus, the allele 15 of D16S539 locus and the allele 11.3 of D8S1179 locus found in a sample from crime scene and it is referred to an unknown person, this indicates that the person is not Egyptian but from Syria.

Conclution: The present data may be considered as a helpful preparation for a preliminary construction of the local DNA database of the Egyptian population .This investigation also recorded an allele specificity for the Egyptian population and provided significant differences in comparison with other neighbouring Arabian ones . Thus , a common STR database for the Arabian populations is a difficult onset . The study also confirmed the efficacy of the BioRobot EZ1 method in DNA extraction yield.

\section{References}

Abdin L., Shimada I., Brinkmann B. and Hohoff C. (2003). Analysis of 15 short tandem repeats reveals significant differences between the Arabian populations from Morocco and Syria. Legal Med., 5: 150-155.
Ashcroft J., Daniels D. J. and Hart S. V. (2002).Using DNA to solve cold cases. NIJ Reports, Special report.

Carvalho S.P.M., Sales-Peres A., Ribeiro-Bicudo L.A.and Alves da Silva R.H. (2010). Quality evaluation of DNA obtained from stored human saliva and its applicability to identification in Forensic Dentistry. Rev. odonto cienc, (25)1: 48-53.

Clayton T.M., Whitaker J.P.and Maguire C.N. (1995). Identification of bodies from the scene of a mass disaster using DNA amplification of short tandem repeat (STR) loci, Forensic Sci. Int. 76 7-15.

ENFSI DNA Working Group: ENFSI survey on DNA databases in Europe, Sept. 2008, www.enfsi.eu/get_doc.php?uid=227. European Council: Resolution of 25 June 2001 on the exchange of DNA analysis results.

Masoud S. (2008). Genetic variation of 15 autosomal short tandem repeat (STR) Loci in the palestinan population of Gaza Strip International Journal of Legal Medicine S150-S195.

Montpetit S.A., Fitch I.T. and O'Donnell P.T. (2005). A Simple automated Instrument for DNA Extraction in Forensic Casework. Journal of Forensic Sciences, vol 50, No 3.

Nedel F., Andre D. A., de Oliveira I.O., Tarquinio S.B. and Demarco F.F (2009): Buccal cells submitted to three different storage conditions before DNA extraction. J Appl Oral Sci,17:113-115.

Phillips H.A., Howard G.C.W. and Miller W.R. . (2000). P53 Mutations As a Marker of Malignancy in Bladder Washing Samples From Patients With Bladder Cancer. Br J Cancer, 82(1): 136-141.

Wallace H.M., Jackson A.R., Gruber J. and Thibedeau A.D. (2014). Forensic DNA databases-Ethical and legal standards: A global review. Egyptian Journal of Forensic Sciences, 4: 57-63.

Wang S.S., Thornton K., Kuhn A.M., Nadeau J.G. and Hellyer T.J. (2003). Homogeneous Real-Time Detection of Single-Nucleotide Polymorphisms by Strand Displacement Amplification on the BD ProbeTec ET System. Clin Chem, 49(10), 1599-1607. 